\title{
Multiple Equilibria as a Difficulty in Understanding Correlated Distributions*
}

\author{
Anirban Kar; Indrajit Ray ${ }^{\ddagger}$ and Roberto Serrano ${ }^{\S}$
}

November 2005

\begin{abstract}
We view achieving a particular correlated equilibrium distribution for a normal form game as an implementation problem. We show, using a parametric version of the two-person Chicken game and a wide class of correlated equilibrium distributions, that a social choice function that chooses a particular correlated equilibrium distribution from this class does not satisfy the Maskin monotonicity condition and therefore can not be fully implemented in Nash equilibrium.
\end{abstract}

JEL Classification Numbers: C72.

Keywords: Correlated Equilibrium Distribution, Implementation, Monotonicity.

${ }^{*}$ We thank Francoise Forges, Chirantan Ganguly and especially Rajiv Vohra for helpful comments. Work on this paper began while Ray was visiting Brown University. Serrano gratefully acknowledges Universidad Carlos III and CEMFI in Madrid for their hospitality, and Fundacion Banco Herrero and Universidad Carlos III for research support.

†Department of Economics, University of Warwick, Coventry CV4 7AL, UK. E-mail: a.kar@warwick.ac.uk; Fax: +44.24.7652.3032.

$\ddagger$ Author for Correspondences. Department of Economics, University of Birmingham, Edgbaston, Birmingham B15 2TT, UK. E-mail: i.ray@bham.ac.uk; Fax: +44.121.414.7377.

$\S$ Department of Economics, Brown University, Box B, Providence, RI 02912, USA. E-mail: Roberto_Serrano@brown.edu; Fax: +1.401.863.1970. 


\section{Introduction}

A correlated equilibrium (Aumann $(1974,1987)$ ) for a normal form game is a Nash equilibrium of an extended game, where the extension consists of a correlation device. The outcomes achieved by this solution concept typically improve upon Nash equilibrium outcomes (Moulin and Vial (1978)). The interpretation of correlated equilibrium requires a (fictitious) mediator; thus it would be desirable to construct a communication process among the players only. Indeed, a recent advancement in the literature (Aumann and Hart (2003), Barany (1992), Ben-Porath (1998, 2003), Forges (1990), Gerardi (2004), Gossner (1998), Gossner and Vieille (2001), Krishna (2005), Lehrer (1996), Lehrer and Sorin (1997), Urbano and Vila (2002)) on communication in games considers the following question: can any correlated equilibrium of a given normal form game be generated as the equilibrium outcome of a communication process among the players? This approach certainly addresses the above desideratum, to the extent that the communication process does not involve the mediator.

A significant body of this literature tries to "implement" a correlated equilibrium of a given game. The typical main result in this literature suggests that the mediator can indeed be replaced. That is, depending on the specific set-up, any correlated equilibrium distribution of a given game (with mild assumptions) can be obtained as a Nash equilibrium outcome of a well-specified communication scheme. In this sense, a correlated equilibrium can therefore be "implemented". The literature also claims, as a corollary to the main theorem, that the set of all correlated equilibria (for a restricted class of games) can also be "implemented".

Although these results are no doubt of importance in our understanding of communication in games and the notion of correlated equilibrium, we argue in this paper that the above mentioned literature fails to highlight a couple of key issues regarding "implementation". These papers do not really appeal to the

notions of the "theory of implementation". Possibly as a result of this, two problems creep in. 
First, the literature does not try to achieve full implementation. That is, it does not address the issue concerning the potential problem of multiplicity of equilibrium. It may be easily conceived that a communication scheme meant to generate one particular correlated equilibrium distribution as an equilibrium outcome may have multiple Nash equilibria leading to different outcomes. Ray (2002) hints at the general difficulty in implementing a direct correlated equilibrium by showing an example where the disobedient strategy profile constitutes an equilibrium that in fact Pareto-dominates the obedient strategy profile and considers non-canonical devices for which the disobedient strategy profile ceases to be an equilibrium.

Second, the way the recent literature claims the whole set of correlated equilibria to be implementable (for example, Corollary 1 in Gerardi (2004)) does not conform with our understanding of the notion of implementation either. The unmediated game forms used to achieve different correlated equilibria are different. To implement the set of correlated equilibria, one should aim to construct a single game form whose set of Nash equilibrium outcomes coincides with the set of correlated equilibrium distributions in question. Clearly, this has not been achieved by the recent literature, although it is of course worth noting that earlier (Barany (1992), Forges (1990)) the research agenda in this area was indeed to search for such a universal mechanism. The recent literature has deviated from this earlier direction, overlooking this important distinction.

This paper appeals directly to the ingredients of the theory of implementation and takes a direct approach to check whether a specific correlated equilibrium distribution can be implemented. We formulate an implementation problem in which the social choice function associates with every payoff matrix (a normal form game) a particular desirable correlated equilibrium distribution of the game and ask whether this function is fully implementable or not. We obtain a negative result. We show that many social choice functions that choose such a correlated equilibrium distribution do not satisfy Maskin monotonicity, and therefore cannot be fully implemented in Nash equilibrium.

We illustrate our approach and the result by considering a parametric ver- 
sion of the two-person Chicken game and a wide class of correlated equilibrium distributions. Our paper provides a precise answer to the problem of full implementation of a particular correlated equilibrium in a wide class - it cannot be achieved. Of course, as follows from the work of Barany (1992) and Forges (1990), the whole set of correlated equilibrium distributions can be implemented by virtue of a universal mechanism. This is a distinction to be highlighted, since it says that while the entire set of correlated equilibrium distributions corresponds to the Nash equilibrium outcomes of a communication mechanism among the players, the same cannot be said about specific correlated equilibrium distributions because of the multiple equilibrium difficulty. For a wide class of correlated equilibrium distributions, no mechanism exists that fully implements them. This suggests a return to the Forges-Barany approach of universal mechanisms in the quest of understanding correlation without mediation.

\section{Implementation}

\subsection{The Game}

Consider the two-player non-cooperative game of Chicken as below, in which each of the two players has two strategies, namely, $A$ and $B$, with $0 \leq a<b<$ $c<d$. Let us also assume that $b+d<2 c$, the importance of which will be made clear in the next subsection.

$$
\begin{array}{ccc} 
& A & B \\
A & a, a & d, b \\
B & b, d & c, c
\end{array}
$$

We shall normalize $a=0$, without loss of generality, in the rest of the paper. This game has two pure Nash equilibria, namely, $(A, B)$ and $(B, A)$, and a mixed Nash equilibrium in which each player plays $A$ with probability $\rho=\frac{(d-c)}{b+(d-c)}$. 


\subsection{The Correlated Equilibrium Distribution}

For the above game (for fixed values of $b, c$ and $d$ ), it will suffice for our purpose to consider the following wide class of correlated equilibrium distributions, a typical element of which is as follows.

$\begin{array}{ccc} & A & B \\ A & 0 & p_{1} \\ B & p_{2} & p_{3}\end{array}$

where, $0<p_{i}<1$, for $i=1,2,3$, and $\sum_{i=1}^{3} p_{i}=1$.

It is easy to check that, for the above distribution to be a correlated equilibrium, we must have $b p_{2}+c p_{3} \geq d p_{3}$ and $b p_{1}+c p_{3} \geq d p_{3}$, i.e., we must have $\rho p_{1}+p_{2} \geq \rho$ and $p_{1}+\rho p_{2} \geq \rho$, where $\rho=\frac{(d-c)}{b+(d-c)}$.

A correlated equilibrium as above will be denoted by $p$, while the set of all such correlated equilibrium distributions will be denoted by $P$. The (expected) payoffs for two players from any $p \in P$ are given by $u_{1}(p)=d p_{1}+b p_{2}+c p_{3}$ and $u_{2}(p)=b p_{1}+d p_{2}+c p_{3}$, respectively.

One may consider the correlated equilibrium distribution that maximizes the sum of the expected payoffs, called the utilitarian correlated equilibrium distribution. Clearly, if $b+d \geq 2 c$, then any convex combination of the two pure Nash equilibrium outcomes of the game generates the utilitarian correlated equilibrium distribution with the sum of the expected payoffs $b+d$. Under the assumption that $b+d<2 c$, we have the following.

Lemma 1 Under the assumption that $b+d<2 c$, the utilitarian correlated equilibrium distribution of the game is $p^{*} \in P$ where $p_{3}^{*}=\frac{1-\rho}{1+\rho}=\frac{b}{b+2(d-c)}$ and $p_{1}^{*}=p_{2}^{*}=\frac{1-p_{3}^{*}}{2}$, i.e., the utilitarian correlated equilibrium distribution of the game is

$$
\begin{array}{ccc} 
& A & B \\
A & 0 & \frac{1-p_{3}^{*}}{2} \\
B & \frac{1-p_{3}^{*}}{2} & p_{3}^{*} \\
\text { where } & p_{3}^{*}= & \frac{1-\rho}{1+\rho}=\frac{b}{b+2(d-c)} .
\end{array}
$$


Proof. Consider the (constrained maximization) problem of maximizing the sum of the expected payoffs, $(b+d)\left(p_{1}+p_{2}\right)+2 c p_{3}$ subject to the constraints, $\rho p_{1}+p_{2} \geq \rho$ and $p_{1}+\rho p_{2} \geq \rho$, where $\rho=\frac{(d-c)}{b+(d-c)}$. Note that the maximand is equal to $2 c-\left(p_{1}+p_{2}\right)(2 c-(b+d))$ and therefore the maximization problem is equivalent to minimizing $\left(p_{1}+p_{2}\right)(2 c-(b+d))$ subject to the above constraints. It is now easy to check that the solution of the above problem is $p_{1}=p_{2}=\frac{\rho}{1+\rho}$ and thus $p_{3}=\frac{1-\rho}{1+\rho}$. Hence, the utilitarian correlated equilibrium distribution is as proposed: $p_{3}^{*}=\frac{1-\rho}{1+\rho}=\frac{b}{b+2(d-c)}$ and $p_{1}^{*}=p_{2}^{*}=\frac{1-p_{3}^{*}}{2}$.

We also observe the following.

Lemma 2 Under the assumption that $b+d<2 c$, for any correlated equilibrium distribution $p \in P, p_{3} \leq p_{3}^{*}=\frac{1-\rho}{1+\rho}=\frac{b}{b+2(d-c)}$.

Proof. Consider any correlated equilibrium distribution $p \in P$. Recall that the sum of the expected payoffs is $(b+d)\left(p_{1}+p_{2}\right)+2 c p_{3}=(b+d)\left(1-p_{3}\right)+2 c p_{3}$. Suppose if possible, $p_{3}>p_{3}^{*}$. Then, as $b+d<2 c$, the sum of the expected payoffs for $p$ becomes higher than that for $p^{*}$, which is a contradiction to Lemma 1. Hence the proof.

\subsection{The Implementation Problem}

Suppose the two players are interested in playing the game according to a particular correlated equilibrium distribution, such as the utilitarian equilibrium (or any other distribution $p \in P$ ). They would like to achieve it, though, as the unique Nash equilibrium outcome of a mechanism that does not contemplate correlation devices. Their problem can then be formulated as an implementation problem.

For any given game of Chicken, as above, we can define an implementation problem as follows. Suppose the designer knows the structure of the Chicken game. However, he does not know the actual payoffs for the two players in the game. Alternatively, suppose the design of the mechanism is made by the two players themselves, who are interested in a mechanism that "works" in order 
to implement the desired distribution for any chicken game. A mechanism is a pair $G=\left(\left(M_{1}, M_{2}\right), g\right)$, where $M_{i}$ is player $i$ 's set of messages, and $g$ : $M_{1} \times M_{2} \mapsto Q$ is an outcome function. (Here, $Q$ denotes the unit simplex over $\{(A, A),(A, B),(B, A),(B, B)\}$.

A payoff profile (not to be used by the mechanism designer, who specifies only outcomes) $\Re$ is described by three numbers,

$$
\Re=\{(b, c, d) \mid 0<b<c<d, \quad b+d<2 c\} .
$$

An outcome in this implementation problem, denoted by $q$, is a probability distribution over the four pure outcomes of the game. We shall use the notation below. $Q$ is the set of all such probability distributions.

$$
\begin{array}{ccc} 
& A & B \\
A & 1-q_{1}-q_{2}-q_{3} & q_{1} \\
B & q_{2} & q_{3}
\end{array}
$$

The players' payoffs (under a profile $\Re$ ) from an outcome $q \in Q$, are simply the expected payoffs with respect to the probability distribution $q$ and are given by $u_{1}^{\Re}(q)=d q_{1}+b q_{2}+c q_{3}$ and $u_{2}^{\Re}(q)=b q_{1}+d q_{2}+c q_{3}$, respectively. The preference over $q \in Q$ for each player $i(i=1,2)$ is denoted by $\succeq_{i}^{\Re}$, under $\Re$, which clearly is defined as, $q \succeq_{i}^{\Re} q^{\prime}$ if and only if, $u_{i}^{\Re}(q) \geq u_{i}^{\Re}\left(q^{\prime}\right)$ for any $q$, $q^{\prime} \in Q$. We will denote the game induced by $G$ with preferences $\left(\left(\succeq_{i}^{\Re}\right)\right)_{i}$ by $G(\Re)$.

A social choice function (SCF) $f$ assigns to each chicken game payoff profile $\Re$ an outcome $f(\Re) \in Q$. An SCF $f$ is said to be Nash implementable if there exists a mechanism $G$ such that for every $\Re$, the unique Nash equilibrium outcome of $G(\Re)$ is $f(\Re)$. The problem that concerns us here is to Nash implement any fixed SCF $f$ that, for each $\Re$, consists of a particular correlated equilibrium distribution: $f(\Re)=p(\Re)=p(b, c, d) \in P$. Recall that, when any $p \in P$ is implemented the players' payoffs are, respectively, $u_{1}(p)=d p_{1}+b p_{2}+c p_{3}$ and $u_{2}(p)=b p_{1}+d p_{2}+c p_{3}$. 


\section{The Main Result}

Maskin (1999) showed that the following monotonicity condition is necessary for Nash implementation of an SCF. An SCF $f$ satisfies Maskin monotonicity if whenever $q \preceq_{i}^{\Re} f(\Re) \Longrightarrow q \preceq_{i}^{\Re^{\prime}} f(\Re)$, for any $q \in Q$, for $i=1,2$, we have $f\left(\Re^{\prime}\right)=f(\Re)$. We shall now prove that no social choice function that assigns $p(\Re)=p(b, c, d) \in P$ satisfies the Maskin monotonicity condition. Therefore, no such SCF can be fully implemented in Nash equilibrium.

Theorem 1 No social choice function that chooses a particular correlated equilibrium distribution $p \in P$ satisfies Maskin monotonicity. Therefore, it cannot be fully implemented in Nash equilibrium.

Proof. For a fixed game of Chicken, let us first fix a particular social choice function given by $f(\Re)=p(\Re)=p(b, c, d) \in P$. Let us denote the corresponding probability of outcome $(B, B)$ by $p_{3}(b, c, d)$. Recall from Lemma 2 that, $p_{3}(b, c, d) \leq p_{3}^{*}(b, c, d)=\frac{b}{b+2(d-c)}$, for any such $(b, c, d)$. To check the Maskin monotonicity condition, we need to consider two profiles such that their lower contour sets are nested and then look at the outcomes that the social choice function chooses at these two profiles.

To prove our result, we shall show that there exists a pair of profiles $\Re$ $(\{(b, c, d) \mid 0<b<c<d$ and $b+d<2 c\})$ and its corresponding $p(b, c, d)$, and $\Re^{\prime}\left(\left\{\left(b^{\prime}, c^{\prime}, d^{\prime}\right) \mid 0<b^{\prime}<c^{\prime}<d^{\prime}\right.\right.$ and $\left.\left.b^{\prime}+d^{\prime}<2 c^{\prime}\right\}\right)$ with its $p\left(b^{\prime}, c^{\prime}, d^{\prime}\right)$, such that the lower contour sets are nested, and however, $p(b, c, d) \neq p\left(b^{\prime}, c^{\prime}, d^{\prime}\right)$.

To do so, first fix any arbitrary profile $\Re_{1}$, given by any arbitrary choice of $\left(b_{1}, c_{1}, d_{1}\right)$ and thereby fix $p_{3}\left(b_{1}, c_{1}, d_{1}\right)$. Now choose a $b_{0}<b_{1}$ sufficiently small

such that $\frac{b_{0}}{b_{0}+2\left(d_{1}-c_{1}\right)}<p_{3}\left(b_{1}, c_{1}, d_{1}\right)$ (one may do so as $\frac{b}{b+2\left(d_{1}-c_{1}\right)}$ is a decreasing function in $b$ ). Now, choose a $c_{0}$ and a $d_{0}$ such that $b_{1}-b_{0}=c_{1}-c_{0}=d_{1}-d_{0}$. Denote the difference $b_{1}-b_{0}$ by $\delta>0$. Denote the profile associated with the numbers $\left(b_{0}, c_{0}, d_{0}\right)$ by $\Re_{0}$.

Consider the lower contour set of $f\left(\Re_{0}\right)$ under $\Re_{0}$ which is the set of distributions $q \in Q$ such that $u_{1}(q) \leq u_{1}\left(p\left(b_{0}, c_{0}, d_{0}\right)\right)$ and $u_{2}(q) \leq u_{2}\left(p\left(b_{0}, c_{0}, d_{0}\right)\right)$. 
This lower contour set is characterized by the following two inequalities:

$$
d_{0} q_{1}+b_{0} q_{2}+c_{0} q_{3} \leq d_{0} p_{1}\left(b_{0}, c_{0}, d_{0}\right)+b_{0} p_{2}\left(b_{0}, c_{0}, d_{0}\right)+c_{0} p_{3}\left(b_{0}, c_{0}, d_{0}\right)
$$

and

$$
b_{0} q_{1}+d_{0} q_{2}+c_{0} q_{3} \leq b_{0} p_{1}\left(b_{0}, c_{0}, d_{0}\right)+d_{0} p_{2}\left(b_{0}, c_{0}, d_{0}\right)+c_{0} p_{3}\left(b_{0}, c_{0}, d_{0}\right)
$$

Now take any $q$ in the lower contour set of $f\left(\Re_{0}\right)$ under $\Re_{1}$, i.e., with $u_{1}(q) \leq$ $u_{1}\left(p\left(b_{0}, c_{0}, d_{0}\right)\right)$ and $u_{2}(q) \leq u_{2}\left(p\left(b_{0}, c_{0}, d_{0}\right)\right)$ under $\Re_{1}$. This lower contour set is characterized by the following two inequalities:

$$
\begin{aligned}
& d_{1} q_{1}+b_{1} q_{2}+c_{1} q_{3} \leq d_{1} p_{1}\left(b_{0}, c_{0}, d_{0}\right)+b_{1} p_{2}\left(b_{0}, c_{0}, d_{0}\right)+c_{1} p_{3}\left(b_{0}, c_{0}, d_{0}\right), \\
& \text { i.e., } d_{0} q_{1}+b_{0} q_{2}+c_{0} q_{3}+\delta\left(q_{1}+q_{2}+q_{3}\right) \\
\leq & d_{0} p_{1}\left(b_{0}, c_{0}, d_{0}\right)+b_{0} p_{2}\left(b_{0}, c_{0}, d_{0}\right)+c_{0} p_{3}\left(b_{0}, c_{0}, d_{0}\right)+\delta \sum_{i=1}^{3} p_{i}\left(b_{0}, c_{0}, d_{0}\right) \\
& \text { i.e., } d_{0} q_{1}+b_{0} q_{2}+c_{0} q_{3} \\
\leq & d_{0} p_{1}\left(b_{0}, c_{0}, d_{0}\right)+b_{0} p_{2}\left(b_{0}, c_{0}, d_{0}\right)+c_{0} p_{3}\left(b_{0}, c_{0}, d_{0}\right)+\delta\left(1-q_{1}-q_{2}-q_{3}\right)
\end{aligned}
$$

and

$$
\begin{aligned}
& b_{1} q_{1}+d_{1} q_{2}+c_{1} q_{3} \leq b_{1} p_{1}\left(b_{0}, c_{0}, d_{0}\right)+d_{1} p_{2}\left(b_{0}, c_{0}, d_{0}\right)+c_{1} p_{3}\left(b_{0}, c_{0}, d_{0}\right), \\
& \text { i.e., } b_{0} q_{1}+d_{0} q_{2}+c_{0} q_{3}+\delta\left(q_{1}+q_{2}+q_{3}\right) \\
\leq & b_{0} p_{1}\left(b_{0}, c_{0}, d_{0}\right)+d_{0} p_{2}\left(b_{0}, c_{0}, d_{0}\right)+c_{0} p_{3}\left(b_{0}, c_{0}, d_{0}\right)+\delta \sum_{i=1}^{3} p_{i}\left(b_{0}, c_{0}, d_{0}\right) \\
& \text { i.e., } b_{0} q_{1}+d_{0} q_{2}+c_{0} q_{3} \\
\leq & b_{0} p_{1}\left(b_{0}, c_{0}, d_{0}\right)+d_{0} p_{2}\left(b_{0}, c_{0}, d_{0}\right)+c_{0} p_{3}\left(b_{0}, c_{0}, d_{0}\right)+\delta\left(1-q_{1}-q_{2}-q_{3}\right)
\end{aligned}
$$

Clearly, any $q$ in the lower contour set of $f\left(\Re_{0}\right)$ under $\Re_{0}$ (satisfying the inequalities 1 and 2) is also in the lower contour set of $f\left(\Re_{0}\right)$ under $\Re_{1}$ (satisfying the inequalities 3 and 4$)$. Thus, the lower contour sets of $f\left(\Re_{0}\right)$ for these profiles are nested. 
However, for Maskin monotonicity to be satisfied, we must have $p\left(b_{1}, c_{1}, d_{1}\right)=$ $p\left(b_{0}, c_{0}, d_{0}\right)$. To show that this is not true, recall that by our choice of $b_{0}$, $\frac{b_{0}}{b_{0}+2\left(d_{1}-c_{1}\right)}<p_{3}\left(b_{1}, c_{1}, d_{1}\right)$. Also, by our choices of $c_{0}$ and $d_{0}, p_{3}^{*}\left(b_{0}, c_{0}, d_{0}\right)=$ $\frac{b_{0}}{b_{0}+2\left(d_{0}-c_{0}\right)}=\frac{b_{0}}{b_{0}+2\left(d_{1}-c_{1}\right)}<p_{3}\left(b_{1}, c_{1}, d_{1}\right)$. Finally, from Lemma 2, we have, $p_{3}\left(b_{0}, c_{0}, d_{0}\right) \leq p_{3}^{*}\left(b_{0}, c_{0}, d_{0}\right)$. Hence, $p_{3}\left(b_{0}, c_{0}, d_{0}\right)<p_{3}\left(b_{1}, c_{1}, d_{1}\right)$. Thus, such a social choice function does not satisfy Maskin monotonicity and therefore cannot be fully implemented in Nash equilibrium.

\section{Remarks}

In this short note, we have argued that the recent literature that considers the problem of implementing a specific correlated equilibrium distribution, rather than the whole set of correlated equilibrium distributions, is flawed in an important way, as the corresponding social choice function may not be fully implemented. We have illustrated our point by using a specific game and a wide class of correlated equilibrium distributions.

Within our set-up, one may still wish to consider subgame-perfect implementation to implement a correlated equilibrium distribution as the unique subgame-perfect equilibrium outcome of a suitably constructed extensive game form.

Also, as is well-known, under very mild conditions, any social choice function can be virtually Nash implemented in the sense that it is possible to implement an outcome that is arbitrarily close to the desired one (Abreu and Matsushima (1992), Abreu and Sen (1991), Matsushima (1988)). Virtual implementation is possible if and only if the condition 'non-empty intersection of lower contour sets' is met. In our context, it is indeed met as we have the outcome $(a, a)$ in the Chicken game. One could thus try to construct a mechanism that will give rise to virtual implementation. This kind of approximation would then provide a way out to the difficulty pointed out here. 


\section{References}

1. Abreu, D. and H. Matsushima (1992), "Virtual implementation in iteratively undominated strategies: complete information," Econometrica, 60, 993-1008.

2. Abreu, D. and A. Sen (1991), "Virtual implementation in Nash equilibrium," Econometrica, 59, 997-1021.

3. Aumann, R. J. (1974), "Subjectivity and correlation in randomized strategies," Journal of Mathematical Economics, 1, 67-96.

4. Aumann, R. J. (1987), "Correlated equilibrium as an expression of Bayesian rationality," Econometrica, 55, 1-18.

5. Aumann, R. J. and S. Hart (2003), "Long cheap talk," Econometrica, 71, 1619-1660.

6. Barany, I. (1992), "Fair distribution protocols or how players replace fortune," Mathematics of Operations Research, 17, 327-340.

7. Ben-Porath, E. (1998), "Correlation without mediation: expanding the set of equilibrium outcomes by cheap pre-play procedures," Journal of Economic Theory, 80, 108-122.

8. Ben-Porath, E. (2003), "Cheap talk in games with incomplete information," Journal of Economic Theory, 108, 45-71.

9. Forges, F. (1990), "Universal mechanisms," Econometrica, 58, 1341-1364.

10. Gerardi, D. (2004), "Unmediated communication in games with complete and incomplete information," Journal of Economic Theory, 114, 104-131.

11. Gossner, O. (1998), "Secure protocols or how communication generates correlation," Journal of Economic Theory, 83, 69-89.

12. Gossner, O. and N. Vieille (2001), "Repeated communication through the mechanism and," International Journal of Game Theory, 30, 41-60. 
13. Krishna, R. V. (2005), "Communication in games of incomplete information: the two-player case," Journal of Economic Theory, Forthcoming.

14. Lehrer, E. (1996), "Mediated talk," International Journal of Game Theory, 25, 177-188.

15. Lehrer, E. and S. Sorin (1997), "One-shot public mediated talk," Games and Economic Behavior, 20, 131-148.

16. Maskin, E. (1999), "Nash equilibrium and welfare optimality," Review of Economic Studies, 66, 23-38.

17. Matsushima, H. (1988), "A new approach to the implementation problem," Journal of Economic Theory, 59, 107-121.

18. Moulin, H. and J. P. Vial (1978), "Strategically zero-sum games: the class of games whose completely mixed equilibria cannot be improved upon," International Journal of Game Theory, 7, 201-221.

19. Ray, I. (2002), "Multiple equilibrium problem and non-canonical correlation devices," Department of Economics, Brown University, Working Paper No. 2002-24.

20. Urbano, A. and J. E. Vila (2002), "Computational complexity and communication: coordination in two-player games." Econometrica, 70, 18931927. 